\title{
Mobile Interaction with the Real World: An Evaluation and Comparison of Physical Mobile Interaction Techniques
}

\author{
Enrico Rukzio ${ }^{1}$, Gregor Broll ${ }^{2}$, Karin Leichtenstern ${ }^{3}$, Albrecht Schmidt ${ }^{4}$ \\ ${ }^{1}$ Computing Department, Lancaster University, UK \\ rukzio@comp.lancs.ac.uk \\ ${ }^{2}$ Media Informatics Group, University of Munich, Germany \\ gregor.broll@ifi.lmu.de \\ ${ }^{3}$ Multimedia Concepts and Applications Group, University of Ausgburg, Germany \\ karin.leichtenstern@informatik.uni-augsburg.de \\ ${ }^{4}$ Fraunhofer IAIS, Sankt Augustin and B-IT, University of Bonn, Germany \\ albrecht.schmidt@iais.fraunhofer.de
}

\begin{abstract}
Mobile devices are more and more used for mobile interactions with things, places and people in the real world. However, so far no studies have discussed which interaction techniques are preferred by users in different contexts. This paper presents an experimental comparison of four different physical mobile interaction techniques: touching, pointing, scanning and usermediated object interaction. To evaluate these techniques across different scenarios and to collect real usage data, four prototypes were implemented: a system for mobile interaction in smart environments, a mobile tourist guide, a mobile museum guide and a prototype for mobile interaction with advertisement posters. In each setting an experimental comparison was performed. Based on the results of these studies, which involved over 60 participants in total, advantages and disadvantages of these interaction techniques are described. Context-specific user preferences are presented for the interaction techniques, to help application designers and developers decide which interaction technique(s) to integrate into their application and which consequences this decision has.
\end{abstract}

Keywords: Physical mobile interaction, touching, pointing, scanning, usermediated object interaction, evaluation, comparison.

\section{Introduction}

An important step towards implementing the vision of ubiquitous computing is the use of mobile devices, which are the first truly pervasive computers and interaction devices. So far, most mobile devices, applications and services mainly focus on the interaction between the user, the mobile device and available services. The context of use is often not considered at all or only marginally. This does not conform to our everyday life and behaviour in which context plays a central role. However, in the last 
few years, a huge interest in industry and academia in using mobile devices for interactions with people, places and things can be observed [1, 2, 3].

This paper coins the term physical mobile interactions to describe such interaction styles in which the user interacts with a mobile device and the mobile device interacts with objects in the real world. They enable the nearly ubiquitous use of mobile services that are connected with smart objects. In the used terminology, a smart object can be a real world object, a person or even a location.

The usage of physical mobile interactions simplifies the discovery and use of mobile services, enables new kinds of object-, person- or location-based applications and removes several limitations of mobile devices. The most important and widespread physical mobile interaction techniques are identified to be touching, pointing, scanning and user-mediated object interaction [4].

But so far very little research is reported that has analyzed which interaction technique should be provided by an application and which interaction technique is preferred by which users in which situation. Because of this, it is very complicated for application designers to decide which physical mobile interaction technique to support within a new application or service. The application context, the location of the object, the distance between object and user, the service related to the object and the capabilities of the mobile device for instance are important factors that influence the preference of a user for a specific type of interaction technique. Therefore a studybased comparison of several types of physical mobile interaction techniques was conducted, with the main focus on an evaluation of which type of interaction technique fits best in which situations, applications and scenarios. Touching, pointing, scanning and user-mediated object interaction were used in four different prototypes and analysed in four different user studies. The results reflect the advantages and disadvantages of these interaction techniques as seen by potential users.

The paper is organized as follows. The next section gives an overview about physical mobile interactions whereby the focus lies on the interaction techniques touching, pointing, scanning and user-mediated object interaction. Next, the prototypes used for the user studies and their implementations are described. We then present the four user studies and the corresponding results. Based on this we summarize the results and discuss the advantages and disadvantages of the different physical mobile interaction techniques in the different contexts. The paper is completed by a discussion and outline of our further work.

\section{Physical Mobile Interactions}

A detailed overview and discussion of physical mobile interaction techniques can be found in $[4,5]$. The aim of this section is primarily to give an introduction into the interaction techniques touching, pointing, scanning and user-mediated object interaction that is needed for the understanding for the following text.

By means of the interaction technique touching the user can select a real world object by touching it with a mobile device or by bringing them close together (e.g. 0 $5 \mathrm{~cm}$ ). Want et al. were one of the first who presented a prototype for this interaction technique which incorporates RFID tags and an RFID reader connected to a mobile 
device, in this case a tablet computer [6]. For instance, they used this prototype to interact with augmented books, documents and business cards to establish links to corresponding services like ordering a book or picking up an email address. In [7] this interaction technique is called TouchMe which is realized via proximity sensors that sense the distance between the augmented object and the mobile device.

By means of the interaction technique pointing the user can select or control a smart object by pointing at it with the mobile device. Fitzmaurice was one of the first who described the concept of using mobile devices for pointing based interactions with smart objects to interact with related services [8]. He described a map on which the user can point to get information about a specific area and an augmented library as a potential application area for this interaction technology. The NaviCam, a mobile device with an attached camera that interprets visual markers on physical objects, was one of the first implementations of this interaction technique [9].

The interaction technique scanning is based on the proximity of mobile device and smart object which can be a real world object as well as a location in general. The mobile device scans the environment for nearby smart objects. This action can be triggered by the user or the environment is permanently scanned by the mobile device. The result is a list of nearby smart objects. The corresponding implementations are also known as location based mobile services. Examples for this are Bluetooth, i-area [10] or the Lancaster Guide project [11].

By means of the interaction technique user-mediated object interaction the user types in information provided by the object to establish a link between them. No special technology is needed to establish a link between the smart object and the mobile device because the user is responsible for this. Examples are portable museum guides where the visitor has to type in a number to get information about an exhibit or a URL printed on an advertisement poster to get access to the corresponding services.

A first analysis of mobile interaction techniques was done by [5]. They focused on the classification of interaction techniques based on previous work and personal experience. In comparison to our work they did not experientially evaluate these techniques, nor did they use questionnaires or user studies to compare the mobile interaction techniques under investigation. Our experience gained with comparing interaction techniques however suggests this is very important to evaluate them with users even if it requires a significant investment in creating prototypes. This is very much in line with the statement reported in [12]: the ultimate test of a product's usability is based on measurements of user's experience with it.

\section{Prototypes}

As already mentioned four prototypes were developed and evaluated. The first subsection describes their purpose and their usage in the user studies. The following subsection then discusses how these prototypes were implemented with different technology. 


\subsection{Purpose and Usage of the Prototypes}

The first prototype mobile interaction in smart environments focuses on the usage of mobile devices for interactions with objects in smart environments [13]. Mobile devices are often seen as control point for smart devices and environments. Similarly mobile devices are regarded in many scenarios as a terminal to access information that is related to real world objects. This includes the provision of additional services, e.g. when the TV is touched the mobile device provides an overview of the programs currently available. A further domain is adding interaction capabilities to devices and things that do not have a user interface, hence acting as a remote control for objects. With this prototype it is possible to use the interaction techniques touching, pointing and scanning to interact with smart objects like a CD player, a radio, heating or a laptop which were augmented by Mifare RFID tags and light sensors. Through this the user is able to receive status information about and to control the devices via the mobile devices. The first row of Table 1 shows how a user touches an augmented CD player. It also presents the hardware used for the implementation of the interaction technique pointing.

The second prototype mobile tourist guide is a mobile guide application through which users can get additional information about exhibits in an art park. This prototype supports the interaction techniques pointing, scanning and user-mediated object interaction for the selection of points of interests. User-mediated object interaction and scanning are interaction techniques that are often used as part of existing mobile outdoor guides. Therefore, the prototype supports these two interaction techniques as well. In addition to those, pointing as a novel interaction technique that requires a short distance to the exhibit is integrated. The exhibits in the park were augmented with information signs showing a number for user-mediated object interaction and a visual code [15] for the interaction technique pointing as can be seen in the first picture of the second row of Table 1.

The third prototype mobile museum guide supports touching, pointing and usermediated object interaction and can be used to get additional information about objects of an exhibition in a museum. User-mediated object interaction is the most typical interaction technique used in such guides and is therefore also supported by this prototype. In addition, touching and pointing are integrated as novel interaction techniques that require a shorter distance to the exhibit. The corresponding user study was conducted in a university building in which part of a train museum was simulated. Therefore several posters showing the name of the exhibit, a picture of it and some textual information were attached to different walls. These posters were augmented with ISO 15693-3 RFID tags, visual codes and numbers. The third row of Table 1 shows how a user touches and points onto the simulated exhibits and a visitor who interacts primarily with the phone when using user-mediated object interaction.

The forth prototype mobile interaction with advertisement posters can be used to order cinema tickets trough the interaction with corresponding augmented advertisement posters [14]. The idea behind this prototype is similar to the previously discussed mobile museum guide. The big difference is that the poster is not just augmented with one link to one service. In this prototype the poster is augmented with multiple tags. There is a visual code beside every Near Field Communication (NFC) sign and on the position of every NFC sign there is a Mifare RFID tag attached to the 
back of the poster. The user can physically click on each of these markers by pointing at or touching them. To buy a movie ticket, for instance, the user has to select the movie, the cinema, the number of persons as well as the desired time slot through touching or pointing at the corresponding parts of the poster.

Table 1. Usage of the interactions techniques provided by the four prototypes.

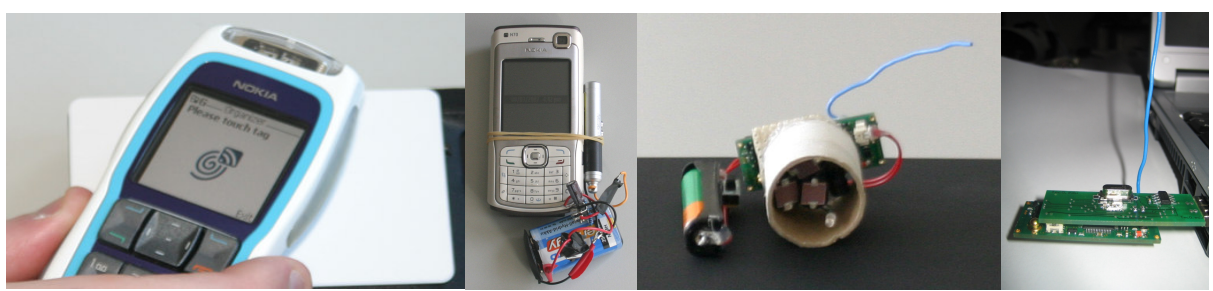

Usage of touching and the hardware for pointing (mobile phone with attached laser pointer, light sensor attached to smart object, USB bridge for receiving the pointing signals) in the first prototype mobile interaction in smart environments

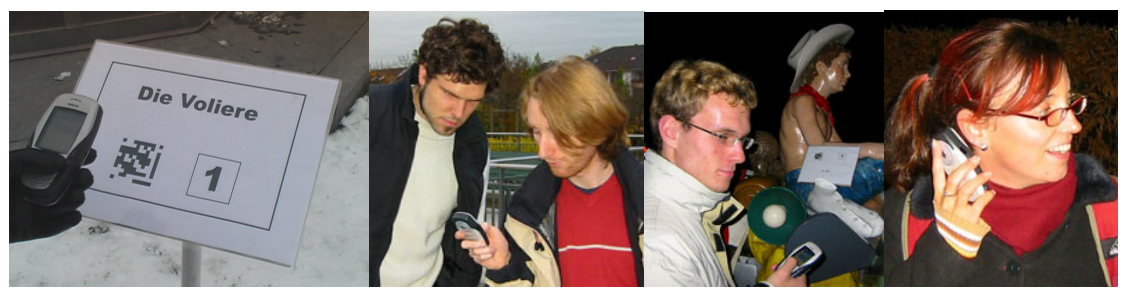

Pictures taking during the user study evaluating the second prototype mobile tourist guide

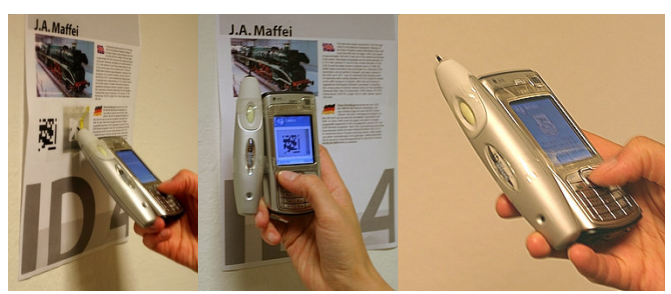

Usage of touching and pointing and user-mediated object interaction the third prototype mobile museum guide

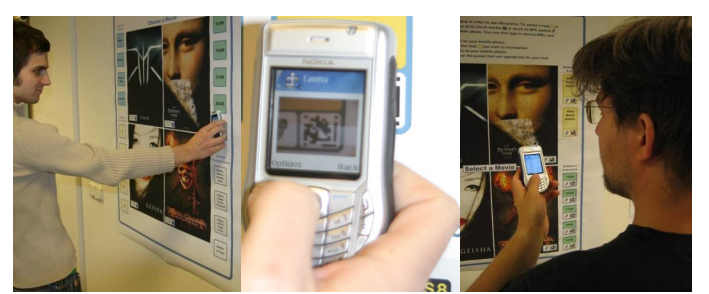

Usage of touching and pointing and user-mediated object interaction in the fourth prototype mobile interaction with advertisement posters 


\subsection{Implementation}

This section discusses the implementation of the four prototypes and the used hardware. Figure 1 shows the underlying basic architecture in which all elements involved in the interaction are depicted: the mobile device, the smart object and related services running on a server. This figure also shows which interaction technique is implemented using which technologies.

Within a physical mobile interaction, the mobile device acts as a mediator between the physical and the digital world. The server represents the digital world which offers information and services related to the smart object. The latter represents the physical world and provides entry points into the digital world. Generally, it can be seen that the smart object provides a link to associated services that are made available by a corresponding server. In all prototypes a person can use her mobile device to interact with a smart object and thus use the service related to the smart object.

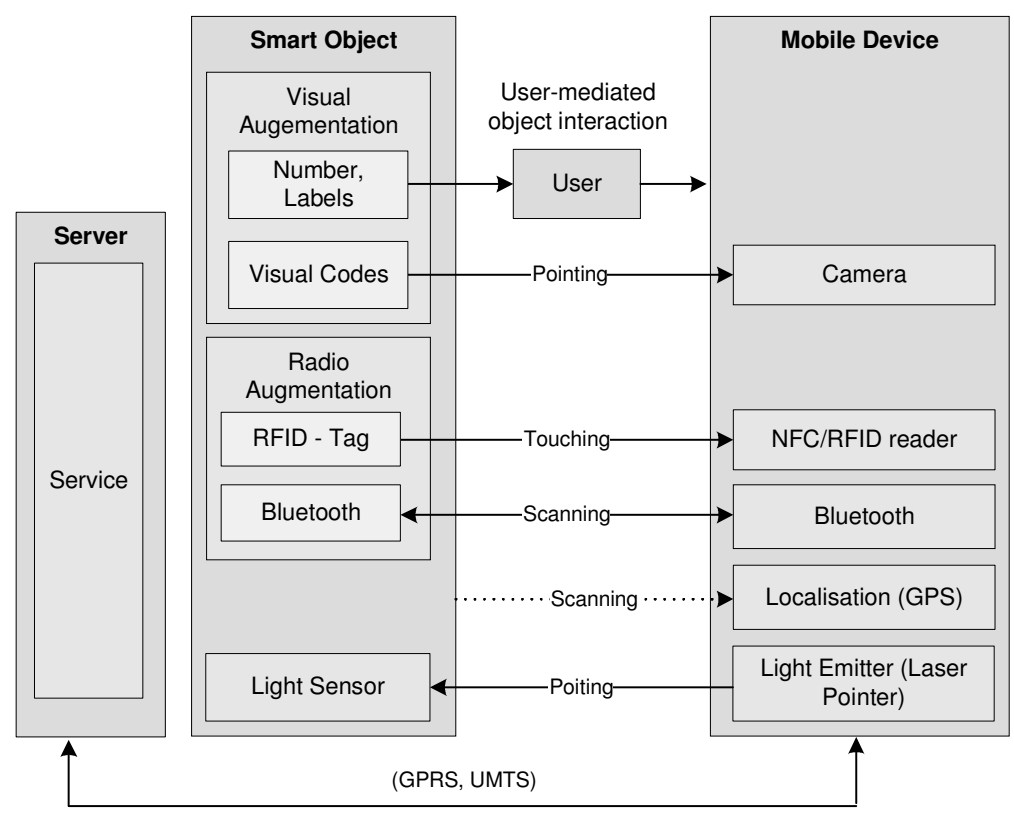

Fig. 1. Basic architecture used for the implementation of the four prototypes.

Table 2 shows the different prototypes, the types of the smart objects, the supported interaction techniques and the used hardware. The table also shows how many persons participated in the user studies, what their average age was and in which month the user study was conducted.

The software running on the mobile device was implemented using Java ME, MIDP 2.0 and CLDC 1.0/1.1. The services running on the server were implemented in Java as well, e.g. using the Java Servlet API and Apache Tomcat. 
Two different devices were used for the implementation of the interaction technique touching. The first implementation is based on a Nokia 3220 with an attached Nokia NFC shell that was used to read Mifare RFID tags. The second one is on the IDBlue RFID pen which was stuck on a Nokia N70 and used to read ISO 15693-3 RFID tags. In this case the smart objects are augmented with RFID tags that can be sensed by mobile devices. Through this the application on a mobile device can read the information stored on the tag and can identify the touched object and the related services.

Two implementations of the interaction technique pointing were used; one was based on a laser pointer and the other on visual codes. The first implementation provides feedback in the real world by using a laser pointer [13] that is physically attached to a mobile phone. The objects which are controlled recognise the beam with light sensors (FW 300) built into the smart object. The recognition algorithm that detects the beam on the light sensor is implemented in a micro-controller that is wirelessly connected to the system. The second implementation uses the built-in cameras of mobile phones to take pictures of visual codes [15]. These are then analyzed and the deciphered information is used to establish a link to the object and the related services.

Table 2. Prototypes, supported interaction techniques, used hardware and information regarding the conducted user studies.

\begin{tabular}{|c|c|c|c|c|}
\hline & $\begin{array}{l}\text { Mobile Interaction in } \\
\text { Smart Environments }\end{array}$ & $\begin{array}{l}\text { Mobile } \\
\text { Tourist Guide }\end{array}$ & $\begin{array}{l}\text { Mobile Museum } \\
\text { Guide }\end{array}$ & $\begin{array}{l}\text { Mobile Interaction with } \\
\text { Advertisement Posters }\end{array}$ \\
\hline $\begin{array}{l}\text { Smart } \\
\text { object }\end{array}$ & $\begin{array}{l}\text { CD player, radio, } \\
\text { heating and laptop }\end{array}$ & $\begin{array}{l}\text { exhibits in an } \\
\text { art park }\end{array}$ & $\begin{array}{l}\text { simulated exhibits } \\
\text { in a museum }\end{array}$ & advertisement poster \\
\hline \multicolumn{5}{|c|}{ Tested physical mobile interaction technique and used hardware respective implementation } \\
\hline Touching & $\begin{array}{l}\text { Nokia } 3220, \text { Nokia } \\
\text { NFC shell, Mifare } \\
\text { RFID tags }\end{array}$ & & $\begin{array}{l}\text { Nokia } 6630 \text { IDBlue } \\
\text { RFID pen, ISO } \\
15693-3 \text { tags }\end{array}$ & $\begin{array}{l}\text { Nokia } 3220, \text { Nokia } \\
\text { NFC shell, Mifare } \\
\text { RFID tags }\end{array}$ \\
\hline Pointing & $\begin{array}{l}\text { Nokia N70, Laser } \\
\text { pointer, light sensors }\end{array}$ & $\begin{array}{l}\text { Nokia 6600, } \\
\text { Visual Codes }\end{array}$ & $\begin{array}{l}\text { Nokia N70, Visual } \\
\text { Codes }\end{array}$ & $\begin{array}{l}\text { Nokia 6630, Visual } \\
\text { Codes }\end{array}$ \\
\hline Scanning & $\begin{array}{l}\text { Nokia N70 } \\
\text { (Bluetooth) }\end{array}$ & $\begin{array}{l}\text { Nokia } 6600, \\
\text { BlueGPS } \\
\text { RBT-3000 }\end{array}$ & & \\
\hline $\begin{array}{l}\text { User- } \\
\text { mediated }\end{array}$ & & $\begin{array}{l}\text { Nokia } 6600, \\
\text { numbers }\end{array}$ & $\begin{array}{l}\text { Nokia N70, } \\
\text { numbers }\end{array}$ & $\begin{array}{l}\text { Nokia } 6630 \text {, labels on } \\
\text { the poster }\end{array}$ \\
\hline \multicolumn{5}{|l|}{ Participants } \\
\hline Number & $\begin{array}{l}20 \\
(35 \% \text { male })\end{array}$ & $\begin{array}{l}17 \\
(77 \% \text { male })\end{array}$ & $\begin{array}{l}8 \\
(88 \% \text { male })\end{array}$ & $\begin{array}{l}17 \\
(77 \% \text { male })\end{array}$ \\
\hline $\begin{array}{l}\text { Average } \\
\text { age }\end{array}$ & 28 & 24 & 28 & 29 \\
\hline Conducted & $03 / 2006$ & $11 / 2005$ & $05 / 2006$ & $06 / 2006$ \\
\hline
\end{tabular}


For the implementation of the interaction technique scanning, the built-in Bluetooth capabilities of mobile phones or external GPS devices were used. The Java ME APIs for Bluetooth (JSR 82) were used to scan for and to connect to other devices. For that purpose, the Bluetooth Serial Port Profile (SPP) of JSR82 which is based on the Bluetooth protocol RFCOMM was used. The GPS-based implementation of scanning used an external GPS device, the BlueGPS RBT-3000 from Royaltek, that can be connected via Bluetooth to a mobile device.

User-mediated object interaction is already available on nearly every mobile phone as it was implemented with standard Java ME interface widgets. The application running on the mobile device simply provides a corresponding input field in which the user types the number or URL she sees on the smart object.

\section{Selected Results of the User Studies}

This section discusses the methodology that was used within all four user studies. After this, every following subsection discusses the results of the evaluation of one prototype and its supported interaction techniques. Only the most relevant, interesting and unexpected results are presented.

\subsection{Methodology}

Each of the following four studies is different regarding the used prototype, the application scenarios and the evaluated interaction techniques. But all of them consisted of three basic steps: a preliminary interview, the use of the prototype and a final interview. The participants were also observed during the study and they were asked to talk about their impressions and experiences while using the prototype. The latter is known as the Thinking Aloud method [16]. Both interviews before and after the study were based on questionnaires including qualitative and quantitative questions. Questions that lead to qualitative results were mostly open questions asking for a general assessment of the interaction techniques or the prototypes itself. Quantitative results are based on predefined answers using a Likert scale. Some questions could also simply be answered with yes or no. The sequence of the usage of the interaction techniques was alternated from user to user to avoid undesired side effects. In some of the following studies the users were asked to rate the interaction technique according to different attributes. At this they could choose between the following possible answers: completely agree (4), partly agree (3), do not know (2), partly disagree (1) and disagree (0). Most of the participants of the user studies were people you often come across in a university building: students, researchers, secretaries, technical staff and visitors.

\subsection{Mobile Interaction in Smart Environment}

The study took place in a domestic home and a smart environment lab, which is a small flat with a bedroom, kitchen, living room and bathroom. During the study the 
participants were sitting in the living room and were asked to accomplish a set of tasks using the prototype. At the beginning of the study the three different interaction techniques were explained to the participants. The users were told that for each task they should select and use the interaction techniques they prefer in the given context.

The first scenario was to operate a CD player. The distance between the user and the device was 3 meters. There were no obstacles between the user and the CD player. 19 of the 20 participants preferred and therefore used pointing. One person used the interaction technique scanning.

In the second scenario the task was to open a webpage that is related to a radio program. The link to the website could be retrieved by selecting the radio. The radio was in close proximity to the user. All participants used the interaction technique touching to retrieve the link. Several users characterized the situation as very convenient for touching. It appeared to most of them that touching is the fastest technique in the setting and physical effort is low because they were in range for touching.

In the third scenario the participants had to operate a device in a different room. They were asked to switch the heating in the bathroom to 25 degree Celsius. All 20 participants in the study used the interaction technique scanning. As going to another room requires significant effort, none of the participant was motivated to move to the other room in order to use pointing or touching.

The last scenario was to access a Wikipedia web page to be displayed on a computer screen that was not in direct reach for the user. There was also no line of sight from the users starting position. In order to use the interaction technique pointing users had to move about one meter. To touch the device the users had to walk about four meters. All of the participants used scanning to select the device.

Overall the experiment showed that the relative location of the user with regard to the device that should be operated is the single most important factor for selecting an interaction technique.

In interviews we confirmed that besides the need for physical activity and interaction speed further factors play a role, most importantly security issues and intuitiveness. It can be summarized that people prefer to touch things that are near and accessible without physical effort. If they are further away and the user has a free line of sight, pointing is the preferred interaction technique. Scanning is used only if the other techniques would require physical effort.

\subsection{Mobile Tourist Guide}

A two-day user-study, in which 17 persons participated, was conducted in the Petuelpark in Munich (Germany) in November 2005. Mostly two participants used the prototype at the same time to simulate the more realistic situation that a small group or a couple is jointly walking through a park and not just a single person on its own. The two participants were asked to interact with six exhibits whereby the prototype was given to the other person after every interaction and every person had to use each of the three supported interaction techniques. After having used the prototype we asked the participants to rate the interaction techniques regarding the attributes simple, enjoyable, innovative and reliable. The corresponding results are depicted in the following Figure 2. 
The participants saw user-mediated object interaction as the simplest interaction technique. Furthermore they saw pointing and scanning as the interaction techniques that were most fun to use whereas user-mediated object interaction with an average rating of 1.3 was seen as the least enjoyable one. This also corresponds to the results regarding the innovativeness of the interaction techniques where the participants equally preferred pointing and scanning to user-mediated object interaction. When looking at the results of the reliability of the interaction techniques then it can be seen that the participants preferred user-mediated object interaction over pointing and scanning.

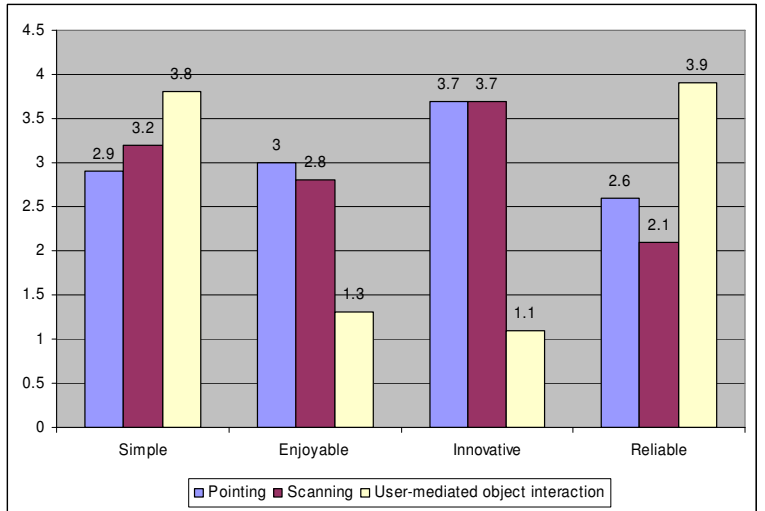

Fig. 2. Average rating regarding the attributes simple, enjoyable, innovative and reliable.

Furthermore we asked them which interaction technique they prefer, they would continuously use and which one they judged to be the most enjoyable, innovative and reliable one. The corresponding results can be seen in Figure 3.

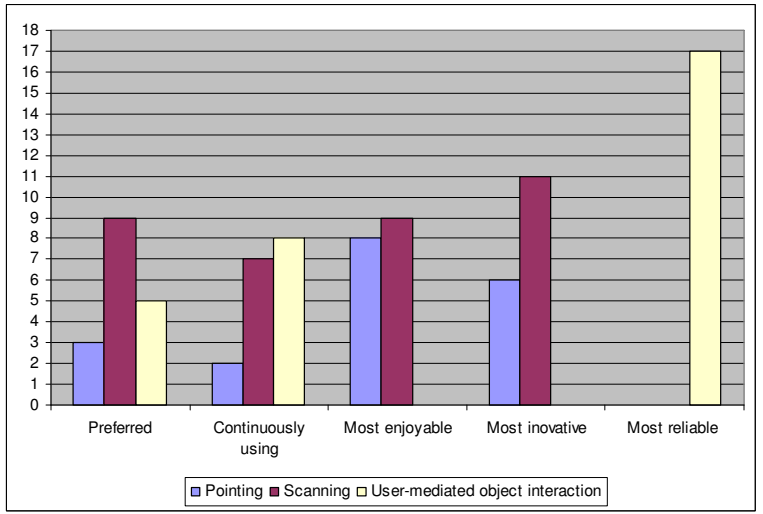

Fig. 3. Preferred ratings of the interaction techniques.

9 of $17(53 \%)$ favoured scanning over user-mediated object interaction (5 of 17 , $29 \%$ ) and pointing (3 of 17, 18\%). These results change a little bit when asking about the interaction technique they would use continuously. Here, user-mediated object 
interaction and scanning were preferred most often whereas pointing was just mentioned by 2 participants. Scanning and pointing were seen as enjoyable and innovative interaction techniques whereas none of the participants connected these attributes with user-mediated object interaction. User-mediated object interaction was unanimously mentioned to be the most reliable technology when comparing it to the others. The reliability results may have been influenced by the problems the participants had with the usage of the implementations of pointing and scanning. The external GPS device sometimes had problems to receive sufficient satellite signals and the accuracy of the identified position of the user was also sometimes not satisfying. When using pointing, some participants had problems to have the marker completely focussed with the built-in camera. Therefore, some of them needed more than one try to successfully use this interaction technique.

\subsection{Mobile Museum Guide}

8 participants used this prototype in order to interact with simulated exhibits in a museum. Every participant used each interaction technique twice whereby the sequence of the used interaction techniques was randomized. After having used the prototype we asked the participants to rate the interaction techniques regarding the attributes simple, enjoyable, innovative and reliable. The corresponding results are depicted in the following Figure 4.

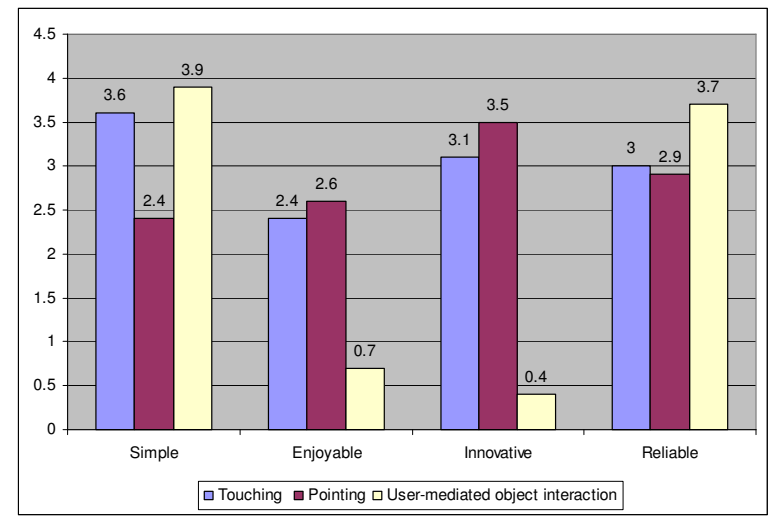

Fig. 4. Average rating regarding the simple, enjoyable, innovative and reliable.

In addition to that, we asked the users what they would rate to be the preferred, most enjoyable, most innovative and most reliable interaction technique. Furthermore, we asked them which interaction technique they would continuously use. The corresponding results are depicted in the following Figure 5.

As one can see when looking at the two figures, pointing and touching are seen as enjoyable and innovative interaction techniques. In contrast to that, user-mediated object interaction and touching are seen as reliable interaction techniques whereby this is not the case when looking at the results of pointing. Furthermore, most participants would prefer the interaction technique touching when continuously using such a system. The reason for that is that a person in a museum is already close to an 
object to perform touching. The most noticeable disadvantage of touching, that the user must be nearby the object, has no impact in this context. Even if the context of a museum was just simulated this study shows the preference of people for touching, their interest in pointing and their trust in user-mediated object interaction.

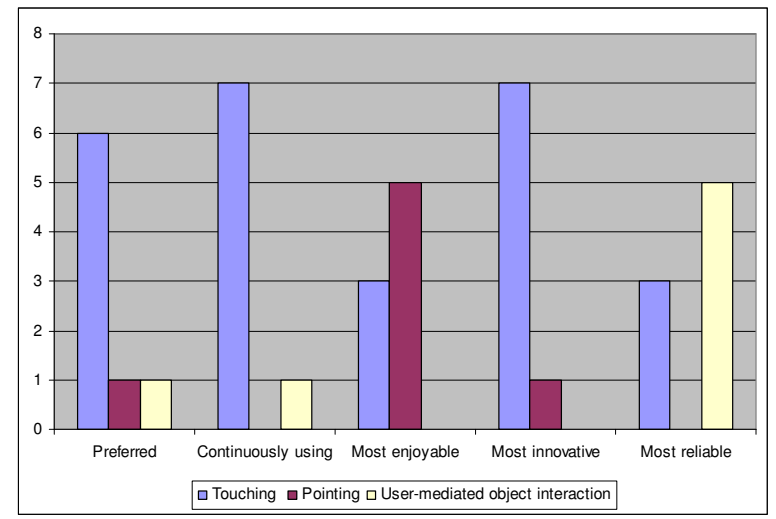

Fig. 5. Preferred ratings of the interaction techniques.

\subsection{Mobile Interaction with Advertisement Posters}

The participants of this user study had to buy a cinema ticket using predefined settings for movie, cinema, number of persons and timeslot. After the usage of the prototype we asked the subjects how easy it is to handle each of the interaction techniques and how enjoyable, innovative and reliable they are. The average of the given answers is depicted by the Figure 6.

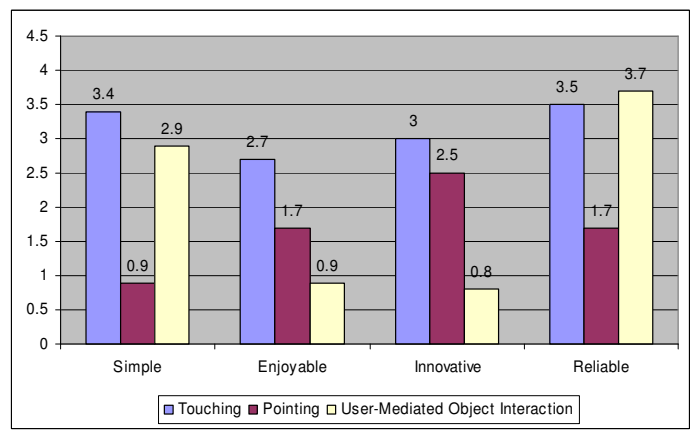

Fig. 6. Average rating of touching, pointing and user-mediated object interaction.

Pointing was not seen as a simple interaction technique because the testers had problems to take a picture of the entire visual code in a sufficient resolution. The results for pointing were in general negatively affected by its implementation that needs a few seconds till the user knows whether she has successfully captured the 
visual code or not. Most testers said that user-mediated object interaction is not an enjoyable or innovative interaction technique. This is probably because people already knew and have already used this interaction technique.

After the user study the participants were asked which interaction technique they preferred and which of the three is the fastest. 13 participants preferred touching and 4 user-mediated object interaction. 12 persons mentioned that touching was the fastest technique whereas 5 mentioned user-mediated object interaction.

When looking at the overall result, touching is seen as the best interaction technique when taking the four analyzed attributes and the questions regarding the preferred and fastest interaction technique into account. Touching was highly ranked in all questions regarding the four attributes easy handling, enjoyable, innovative and reliable. User-mediated object interaction is seen as a reliable interaction technique that is easy to handle but is not enjoyable or innovative. Pointing received the worst marks but is seen as more innovative and enjoyable than user-mediated object interaction.

\section{Advantages and Disadvantages of the Different Physical Mobile Interaction Techniques}

This section summarises the results of the four studies described in the previous section. These results can help application designers and developers when deciding which physical mobile interaction technique should be supported within their application and which consequences a corresponding decision has. In the following, the properties of each interaction technique under evaluation are recapitulated and a set of findings is derived suggesting the use of specific techniques under specific circumstances.

\subsection{Touching}

Touching is regarded as an intuitive, very quick, enjoyable, reliable, unambiguous, innovative, simple and secure interaction technique which potentially requires physical effort but requires only little cognitive load.

It is seen as an intuitive interaction technique because of its directness and the similarity to real world activities such as pressing a button on the microwave. The interaction consists mostly of touching the smart object, waiting for feedback that the touching was successful and recognising the feedback provided by the mobile device.

This interaction technique is also very error resistant when compared to pointing or scanning. The studies have shown that people can learn touching very fast and that they make very few errors after they are used to it. It would be an error, for instance, if the NFC/RFID tag is touched too briefly so that the mobile device can not read it.

It is also an unambiguous interaction technique because first of all, it is technically impossible to read two tags at the same time in the described prototypes and second, because of the directness it is hardly possible to select a wrong tag or smart object. 
Touching is typically preferred when the smart device is in reach of the user. Touching often requires the users' motivation to approach the smart device. But people try to avoid this physical effort, especially when being at home. The user studies based on prototypes of a mobile museum guide and mobile interaction with advertisement posters shows that in these cases touching is the preferred interaction technique. In these situations the user is anyway interested in being near the smart object and in this situation many of them prefer touching because of the previously mentioned advantages.

\subsection{Pointing}

Pointing is seen as an innovative, enjoyable and intuitive technique that requires some cognitive effort to point at the smart device and needs line of sight. It is typically preferred when the smart device and its tag are in line of sight of the user and the smart device cannot be accessed directly by the user. In the users' minds, pointing makes most sense because it combines an intuitive interaction with less physical effort (no need to actually approach the object in question).

It is also seen as an intuitive interaction technique, because it corresponds to our everyday behaviour to point at things when talking about them. Furthermore, it is an example of direct interaction techniques; these are generally seen as simpler as indirect interaction techniques.

When being at interaction distance, pointing is seen as the quicker interaction technique when comparing it to scanning but it is considered to be slower than touching. Fastness, error resistance and required physical effort of the interaction technique pointing depend heavily on its implementation. Both implementations discussed previously have in common that they require some dexterity to correctly point at the light sensor or the visual marker.

When using a laser pointer, as in the study described in subsection 4.2 , it can be seen as a fast and simple type of interaction that consists only of the pointing task, waiting for the confirmation of the interaction and getting. The error resistance of this implementation is also high because the user gets direct feedback whether the interaction was recognized and whether the correct object was selected.

In all other studies, described in subsections 4.3, 4.4 and 4.5, a Java-based implementation using visual markers was used. Here the testers did not get a rapid feedback. First they had to take a picture of the marker, then this had to be analyzed by the Java version of the visual code software and then the user gets a feedback about its success or failure. Because of this, it takes several seconds until the user knows whether the visual code was recognized or not. Therefore, this implementation of the interaction technique pointing is not fast and also not error resistant. The latter especially results from the delay between taking a picture and getting the information that the image of the marker was not recognized.

Furthermore the used mobile phones and the size of the used marker also limited the distance in which an interaction was possible. This will change in the future, as mobile phones will have cameras with a high resolution and an optical zoom. The allowed distance is also based on the size of visual markers which was relatively small in presented prototypes. 
A disadvantage of this interaction technique is the coordinative effort and cognitive load to point the mobile device to the marker or light sensor on the smart object. Pointing with the laser on a sensor and taking a picture of a visual marker needs considerable concentration and physical skills, especially from inexperienced users.

\subsection{Scanning}

Scanning is seen as an innovative, somewhat enjoyable and very technical interaction technique which is more complex to use because of its indirectness. Therefore the indirect mobile interaction technique scanning is avoided in many cases. If there is line of sight, the user normally switches to a direct mobile interaction technique such as touching or pointing.

Indirect interaction is mainly used to bridge a physical distance and to avoid physical effort. Scanning is seen as the technique with the least physical effort. Users tend to switch to scanning if a movement would be necessary to use a direct interaction technique.

A disadvantage is, that the user has to select the intended device when using for instance a Bluetooth based implementation of this interaction technique; this process is more time-consuming than directly interacting when standing close to a smart object. Furthermore the cognitive effort is higher compared to pointing or touching. It is typically used when the smart device and its tag can not be seen by the user and when the smart device is in scanning range.

A further advantage of scanning is the possibility to get a list of all smart objects in the vicinity. Thus it can be avoided to miss one. Additionally, no visual augmentation to attract the attention of the user is needed.

A disadvantage of scanning is that the user has to establish the mapping between an item on the list or map presented by the mobile device and the objects in the environment for which a high cognitive effort is required. This might sometimes lead to frustration or the interaction of the wrong object.

The study described in subsection 4.3 showed, that many testers saw the interaction technique scanning as a technique which they would prefer when using a mobile tourist guide. One important reason for this was that they like to get proactively informed when a sight is nearby.

The presented studies were conducted using on two different implementations of the interaction technique. One was based on Bluetooth and one on GPS. When using Bluetooth then the users did not like the time which is needed to show a list of nearby objects. The disadvantage of GPS - which was seen by the testers - was that the GPS device had sometimes problems to deliver the exact position on time.

\subsection{User-mediated Object Interaction}

User-mediated object interaction is seen as a very reliable and simple interaction technique. The user has to establish a link between a smart object and a mobile service. In the previously discussed user studies (e.g. section 4.2 and 4.3) this merely meant typing in a simple number. 
This view on simplicity and reliability might change when the user has to copy a URL using T9. This is much more cumbersome and the possibility of typing in a wrong URL is much higher. The performance of this interaction technique depends also on length of the information which has to be typed in. User-mediated object interaction is relative fast when the user has to type in for instance a three digit number and is relative slow when the user has to type in a long URL.

User-mediated object interaction is well known because it is, in contrast to the others, already used in mobile guides and many people already have experiences with its usage. Mainly because of this it is not seen as a enjoyable or innovative interaction technique.

\subsection{Summary}

The following Table 3 shows the advantages and disadvantages of the analyzed physical mobile interaction techniques based on the findings discussed in the previous subsections. This table also discusses attributes like fun factor or innovativeness which might be a reason for a potential costumer to buy or use a mobile service or application.

Table 3. Comparison of properties of the touching, pointing and scanning.

\begin{tabular}{|c|c|c|c|c|}
\hline & Touching & Pointing & Scanning & User-mediated \\
\hline \multicolumn{5}{|c|}{ Rating: Good, Average, Bad } \\
\hline $\begin{array}{l}\text { Felt error } \\
\text { resistance, } \\
\text { reliability }\end{array}$ & Good & $\begin{array}{l}\text { Good (laser } \\
\text { pointer) - } \\
\text { Bad (visual } \\
\text { marker) }\end{array}$ & Average & $\begin{array}{l}\text { Good (short } \\
\text { number) - Average } \\
\text { (long number) }\end{array}$ \\
\hline $\begin{array}{l}\text { Performance, speed } \\
\text { (within interaction } \\
\text { distance) }\end{array}$ & Good & Average & $\begin{array}{l}\text { Bad (Bluetooth) - } \\
\text { Good (GPS) }\end{array}$ & $\begin{array}{l}\text { Average (short } \\
\text { number) - Bad } \\
\text { (long number) }\end{array}$ \\
\hline $\begin{array}{l}\text { Simplicity, } \\
\text { Intuitiveness }\end{array}$ & Good & $\begin{array}{l}\text { Good (laser } \\
\text { pointer) - Bad } \\
\text { (visual marker) }\end{array}$ & $\begin{array}{l}\text { Average (GPS) - } \\
\text { Average } \\
\text { (Bluetooth) }\end{array}$ & $\begin{array}{l}\text { Good (short } \\
\text { number) - Average } \\
\text { (long number) }\end{array}$ \\
\hline \multicolumn{5}{|c|}{ Rating: High, Medium, Low } \\
\hline Cognitive load & Low & $\begin{array}{l}\text { Medium (laser } \\
\text { pointer) } \\
\text { - High (visual } \\
\text { marker) }\end{array}$ & $\begin{array}{l}\text { High (Bluetooth) - } \\
\text { Medium (GPS) }\end{array}$ & $\begin{array}{l}\text { Medium (short } \\
\text { number) - High } \\
\text { (long number) }\end{array}$ \\
\hline Physical effort & High & Medium & Low & Low \\
\hline Fun factor & High & High & $\begin{array}{l}\text { High (GPS) - } \\
\text { Medium } \\
\text { (Bluetooth) }\end{array}$ & Low \\
\hline Innovativeness & High & High & High & Low \\
\hline
\end{tabular}




\section{Conclusion}

In this paper we have presented a comprehensive experimental evaluation and comparison of the physical mobile interaction techniques touching, pointing, scanning and user-mediated object interaction. Therefore four different user studies were conducted in which the interaction techniques were evaluated in the context of mobile interaction in smart environments, a mobile tourist guide, a mobile museum guide and mobile interaction with advertisement posters.

The results show that in a smart environment the distance between the user and the smart object is an important factor for the preference of an interaction technique. If the object is within grasp, users prefer touching, if the user is too far away for touching but there is a line of sight, users prefer pointing, and in all other cases they prefer scanning. This is not true for the context of a mobile museum and tourist guide in which the user is interested in a nearby exhibit or sight anyway. Therefore, the distance between object and user does not play an important role. In this setting, factors like proactive behaviour of the application supported by scanning, simplicity and reliability provided by user-mediated object interaction, innovativeness and fun aspects related with touching, pointing and scanning or simplicity provided by touching and user-mediated object interaction can lead to user preference in a given context. The results presented here regarding the advantages and disadvantages seen by the users can be used by application designers when deciding which interaction technique(s) should be provided by their mobile application.

In our future work we will investigate further interaction techniques, other implementations of them and more applications contexts. We also plan to conduct long term studies in practical contexts to learn more whether and how the preferences of the users for the interaction techniques change over time. The findings provide grounding for creating adaptive user interfaces, that take location and activity into account. In further research we investigate how such adaptive user interfaces can be designed to enable efficient and effective interaction.

\section{Acknowledgement}

Important parts of the presented research (especially the prototype about mobile interaction with advertisement posters) were performed in the context of the Perci (PERvasive serviCe Interaction) project [17] which was funded by DoCoMo EuroLabs.

\section{References}

[1] Tim Kindberg; John Barton; Jeff Morgan; Gene Becker; Debbie Caswell; Philippe Debaty; Gita Gopal; Marcos Frid; Venky Krishnan; Howard Morris; John Schettino; Bill Serra; Mirjana Spasojevic: People, places, things: web presence for the real world. In: Mobile Networks and Applications, 7 (5), pages 365--376, ISSN 1383-469X, 2002. 
[2] John Boyd: Here Comes The Wallet Phone. In: IEEE Spektrum, 42 (11), pages 12-13, 2005.

[3] Geoffrey A. Fowler: QR codes: In Japan, Billboards Take Code-Crazy Ads to New Heights. In: Wall Street Journal, 10.10.2005, 2005.

[4] Enrico Rukzio. Physical Mobile Interactions: Mobile Devices as Pervasive Mediators for Interactions with the Real World. PhD Dissertation. Faculty for Mathematics, Computer Sience and Statistics. University of Munich. 2006.

[5] Rafael Ballagas; Michael Rohs; Jennifer Sheridan; Jan Borchers: The Smart Phone: A Ubiquitous Input Device. In: IEEE Pervasive Computing, 5 (1), pages 70-77, 2006.

[6] Roy Want; Kenneth P. Fishkin; Anuj Gujar; Beverly L. Harrison: Bridging physical and virtual worlds with electronic tags. Proceedings of the SIGCHI conference on Human factors in computing systems: the CHI is the limit, ACM Press, ISBN 0-201-48559-1, Pittsburgh, Pennsylvania, United States, 1999.

[7] Pasi Välkkynen; Ilkka Korhonen; Johan Plomp; Timo Tuomisto; Luc Cluitmans; Heikki Ailisto; Heikki Seppä: A user interaction paradigm for physical browsing and near-object control based on tags. Physical Interaction (PI03) - Workshop on Real World User Interfaces in conjunction with Mobile HCI 2003, Udine, Italy, 2003.

[8] George W. Fitzmaurice: Situated information spaces and spatially aware palmtop computers. In: Communications of the ACM, 36 (7), pages 39-49, ISSN 0001-0782, 1993.

[9] Jun Rekimoto; Katashi Nagao: The World Through the Computer: Computer Augmented Interaction with Real World Environments. Proceedings of the 8th ACM Symposium on User Interface Software and Technology (UIST '95), pages 29-36, Pittsburgh, PA, USA, 1995.

[10] NTT DoCoMo i-area: Location Based Services, http://www.nttdocomo.co.jp/english/service/imode/site/i_area.html

[11] Keith Cheverst; Nigel Davies; Keith Mitchell; Adrian Friday; Christos Efstratiou: Developing a context-aware electronic tourist guide: some issues and experiences. Proceedings of the SIGCHI conference on Human factors in computing systems, ACM Press, ISBN 1-58113-216-6, The Hague, The Netherlands, 2000.

[12] Alan Dix; Janet Finlay; Gregory D. Abowd; Russell Beale: Human Computer Interaction. 3rd Edition, Prentice Hall, ISBN 0130461091, 2003.

[13] Enrico Rukzio; Karin Leichtenstern; Vic Callaghan; Paul Holleis; Albrecht Schmidt; Jeannette Chin: An Experimental Comparison of Physical Mobile Interaction Techniques: Touching, Pointing and Scanning. Eighth International Conference on Ubiquitous Computing (Ubicomp 2006), Orange County, California, 2006.

[14] Gregor Broll; Sven Siorpaes; Enrico Rukzio; Massimo Paolucci; John Hamard; Matthias Wagner; Albrecht Schmidt: Supporting Mobile Service Usage through Physical Mobile Interaction. Fifth Annual IEEE International Conference on Pervasive Computing and Communication (PerCom 2007), White Plains, NY, USA, 2007.

[15] Michael Rohs; Beat Gfeller: Using Camera-Equipped Mobile Phones for Interacting with Real-World Objects. In: Advances in Pervasive Computing, Austrian Computer Society (OCG) pages 265-271, ISSN 3-85403-176-9, 2004.

[16] Clayton Lewis; John Rieman: Task-Centered User Interface Design: A Practical Introduction. http://www.hcibib.org/tcuid/, 1994.

[17] Perci project, http://www.hcilab.org/projects/perci/ 